\title{
Implications for present and future applications of the implantable cardioverter-defibrillator resulting from the use of a simple model of cost efficacy
}

\author{
M H Anderson, A J Camm
}

\begin{abstract}
Objective-To develop a model to assess the cost-efficacy of the implantable cardioverter defibrillator to prevent sudden death. The model must be sufficiently flexible to allow the use of cost and survival figures derived from different sources.
\end{abstract}

Setting-The study was conducted in a teaching hospital department of cardiology with experience of 40 implantable cardioverter defibrillator implants and a large database of over 500 survivors of myocardial infarction.

Procedure-The basic costs of screening tests, stay in hospital, and purchase of implantable cardioverter defibrillators were derived from St George's Hospital during 1991. To assess the cost-efficacy of various strategies for the use of implantable cardioverter defibrillators, survival data taken from published studies or from our own database. Implications of the national cost of the various strategies were calculated by estimating the number of patients a year requiring implantation of a defibrillator if the strategy was adopted.

Results-Use of implantable cardioverter defibrillators in survivors of cardiac arrest costs between $£ 22400$ and $£ 57000$ for each year of life saved. Most of the strategies proposed by the current generation of implantable cardioverter defibrillator trials have cost efficacies in the same range, and adoption of any one of these strategies in the United Kingdom could cost between $£ 2$ million and $£ 100$ million a year. Future technical and medical developments mean that costefficacy may be improved by up to $80 \%$. Due to the limitations of screening tests currently available restriction on the use of implantable cardioverter defibrillators to those groups where it seems highly cost-effective will result in a small impact on overall mortality from sudden cardiac death.

Conclusion-Present and possible future applications of the implantable cardioverter defibrillator seem expensive when compared with currently accepted treatments. Technical and medical developments are, however, likely to result in a dramatic improvement in cost efficacy over the next few years.

\section{(Br Heart $\mathcal{~} 1993 ; 69: 83-92)$}

Since the first implant in humans of an implantable cardioverter defibrillator (ICD) in $1980^{1}$ the number of devices implanted annually has almost doubled each year. ${ }^{2}$ By the end of 1991 over 20000 devices had been implanted worldwide ${ }^{3}$ with probably $80 \%$ of these implants occurring in the United States. In 1990 the total number of patients who had received an ICD in the United Kingdom was $40 .{ }^{4}$ This has currently risen to between 160 and 200. These figures suggest an implantation rate of around $5 \%$ of that of the United States.

As with many other new medical technologies this dramatic explosion in use has occurred before conclusive evidence of the efficacy of the treatment is available. The reasons for this are not hard to find. There is no question that the ICD is capable of reliable defibrillation. What is less clear is what impact this has on overall patient survival and quality of life. Pending the availability of the results from several randomised controlled trials that are in progress the results of historical and non-controlled comparisons suggest that the device is effective in reducing mortality from sudden cardiac death. ${ }^{5-8}$

One factor that has limited the more widespread use of the ICD within the United Kingdom has been the high initial cost of the device $(£ 10000-£ 18000)$. The ICD has been perceived as an expensive treatment because of the capital cost of this single item, which cannot easily be accommodated within existing budgets. This may, however, represent an unfair comparison with other treatments where the total cost may be similar but more widely dispersed.

Organised attempts to assess the costefficacy of medical interventions are rare. In the light of the perceived expense of ICD treatment, however, four groups have considered its cost-efficacy. Kupperman and colleagues produced a figure of $\$ 17400$ for each life-year saved (1986 prices) but their model suggested this could fall to $\$ 7400$ per life-year by 1991 as a result of increased working life of defibrillators and reduced admissions to hospital. ${ }^{9}$ These figures suggested that the 
Table 1 Assessment of cost-efficacy of the ICD. Kupperman et al. ${ }^{9}$ (1986 prices)

\begin{tabular}{ll}
\hline Procedure & $\begin{array}{l}\text { Cost-efficacy } \\
\text { ( } \text { per life-year) }\end{array}$ \\
\hline Hospital haemodialysis & 59500 \\
Coronary artery bypass grafting for severe & 44200 \\
angina (single vessel disease) & \\
Heart transplantation & 26900 \\
Treatment for mild hypertension & 23200 \\
ICD (1986) & 17400 \\
Treatment for severe hypertension & 11100 \\
ICD (1991) & 7400 \\
Coronary artery bypass grafting for 3 & 7200 \\
$\quad$ vessel disease & \\
\hline
\end{tabular}

ICD is equivalent in costefficacy to many other medical treatments (table 1).

In the United Kingdom Buxton and coworkers have used a similarly complex model to assess the cost-efficacy of the ICD in comparison with long-term treatment with amiodarone. ${ }^{10}$ Their model studied a 20 year period with extrapolated survival data from various studies, and produced a cost-efficacy ranging from $£ 10000$ to $£ 20000$ per life-year falling with technical and implantation developments to as low as $£ 6000$ per lifeyear. ${ }^{3911}$

In a simple study that considered only the in hospital costs of ICD use O'Donoghue and colleagues have shown a $20 \%$ saving associated with early implantation of the ICD when compared with more extensive evaluation of antiarrhythmic drug treatment. ${ }^{12}$ The overall cost-efficacy of these different strategies was not considered.

Most recently Larsen et al used a Markov state transition model to compare the costefficacy of the ICD with that of treatment with amiodarone and produced a marginal cost-effectiveness of $\$ 29200$ per life-year for the ICD compared with treatment with amiodarone. ${ }^{13}$

Although they are comprehensive approaches to the assessment of ICD costefficacy none of the four studies described provide a simple method to assess the costefficacy of the ICD with different survival data or costs. They highlight the need for a simple, flexible, cost-efficacy model that may be used to assess new and existing strategies for the use of implantable defibrillators. Such a model should be capable of using locally derived costs and survival data to maximise the relevance of its output.

The aim of this study was to develop a model that could be used to assess the costefficacy of the ICD in various clinical situations and that was sufficiently adaptable to be used by other workers to assess the impact of local costs and survival figures. With this model we aimed to assess the cost-efficacy of present and future applications of the ICD and the possible impact of technical developments on cost-efficacy.

\section{Method}

The calculation of cost-efficacy used in our model is based on the simple equation:

$$
\text { Cost-efficacy }=\frac{\text { total cost of use of ICD in a group }}{\text { gain in years of life in the group }}
$$

Cost efficacy is expressed as cost (in pounds sterling) per life-year $(£ / 1-y)$. This is the amount of money that must be spent to give one patient one extra year of life. No adjustment is made for the quality of this life as only a few small studies have so far consid- 0 ered this aspect of the use of ICDs. ${ }^{14-16}$ Because of the limited availability of longterm survival data for recipients of ICDs a fixed time period has been used for the calculation. This period is assumed to be three $\frac{\overrightarrow{\bar{S}}}{\vec{F}}$ years except where otherwise stated.

In calculating the total cost of use of ICDs 흠 we take the cost of identifying the patients at $\frac{\bar{\rho}}{\frac{1}{\sigma}}$ risk (screening tests), the cost of the hospital $\underset{\Omega}{\Phi}$ stay required for implantation of the ICD, the cost of the implantation surgery, the cost of $\vec{\theta}$ the ICD generator and leads, and the cost of ${ }_{-}^{\circ}$ follow up over the life of the generator. These $\vec{\omega}$ costs are written off over the three year period of the study. Subsequent costs of replacement? of the generator are not considered.

To calculate the gain in years of life $\overrightarrow{-}$ accruing from the use of the ICD in any par- $\stackrel{\omega}{\circ}$

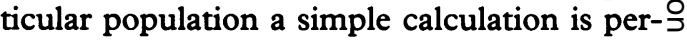
formed. Figure 1 shows the survival curve for a hypothetical population of 100 subjects in whom the sudden death is $16 \%$ in the first year, $8 \%$ in the second year, and $4 \%$ in the third year. If the defibrillator prevents all the $\vec{\varphi}$ sudden deaths over the three year predicted $\Theta$ life of the ICD generator then 16 patients 0 who would have died during the first year will gain a mean additional survival of $2 \cdot 5$ years (assuming sudden deaths are evenly distributed throughout the year) giving a total gain $\frac{}{\odot}$ of 40 life-years. A similar calculation can be $\underset{\vec{P}}{\overrightarrow{0}}$ performed for the second and third years. $\frac{3}{3}$ Patients who die as a result of implantation of $\vec{P}$ an ICD actually lose life-years and this must also be taken into account. Their death also reduces the number of life-years gained byฏ the rest of the population. Knowing the cost for 100 defibrillator implants and follow up over the three year period and the net gain in life-years we can calculate the cost per life-year.

Table 2 shows the cost assumptions that have been used in our model. These are

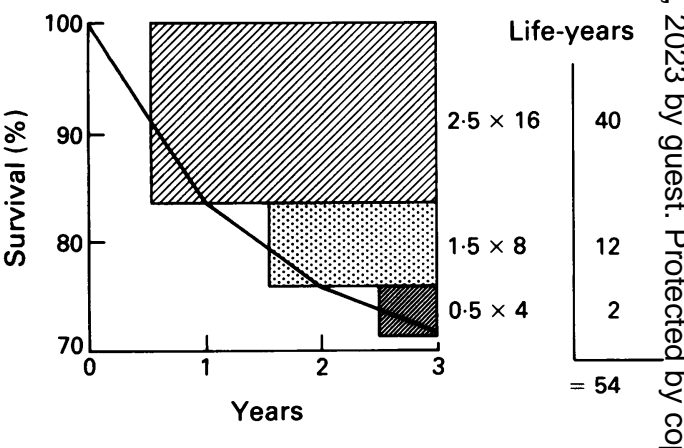

Figure 1 Method of calculation of gain in life-years. The dark line shows the untreated survival free of sudden death in a hypothetical population of 100 patients. The shaded boxes represent the gain in life-years accruing if the ICD prevents all sudden deaths. The total gain of 54 life-years calculated is reduced to 52.4 years $(54 \times(100-3)$ as the $3 \%$ of the population who die gain no benefit from the ICD. Also the three patients who die lose nine life-years so the final gain from the strategy is 43.4 life-years. 
Table 2 The cost assumptions used in our model (1991 prices)

\begin{tabular}{lr}
\hline Item & Cost $(£)$ \\
\hline Screening tests: & \\
Echocardiogram & 55 \\
Signal average electrocardiogram & 50 \\
Holter recording and analysis & 100 \\
Limited electrophysiological study & 500 \\
$\quad$ (stimulation of ventricular tachycardia) & 150 \\
Repeat ventricular tachycardia stimulation study & 10000 \\
ICD generator & 1650 \\
ICD leads (including patches and pace and & 3200 \\
sense leads) & 265 \\
ICD implantation surgery cost ${ }^{10}$ & 100 \\
Additional hospital stay (charge/day) & \\
Follow up visit (six in 1st, four in other years) &
\end{tabular}

based on 1991 prices and have been derived from several sources. The single most expensive item at any ICD implant is the ICD generator itself. The purchase cost of an ICD generator in the United Kingdom is currently from $£ 5000$ for a simple defibrillation only device without Holter or datalogging functions to $£ 15000$ for a third generation device with bradycardia support pacing, antitachycardia pacing, low energy cardioversion, and datalogging facilities. The cost of ICD models can vary considerably depending on the manufacturer's view of their current position within the market. The figure of $£ 10000$ that we have used in our model allows the purchase of a modern device without antitachycardia pacing but with datalogging and Holter functions. The ICD also requires the purchase of pace and sense electrodes and epicardial patches (two or three) or transvenous electrode(s) with or without an axillary patch electrode. Although the costs of individual components of these systems vary considerably between manufacturers the total cost of a complete lead system is remarkably similar. Our figure of $£ 1650$ reflects the cost (at 1991 prices) of the electrodes used in the first 14 epicardial implants performed at this hospital.

Duration of surgery for epicardial ICD implantation has been similar to that for coronary artery bypass surgery and though the amount of disposable equipment used at ICD implantation is less the additional cost of technicians and cardiology staff means that the cost of each operation is similar. We have not attempted a detailed cost analysis of ICD implantation surgery but have taken a figure of $£ 3200$ for surgical implantation with epicardial patches. This figure represents the 1991 cost of a coronary artery bypass opera-

Table 3 The cost efficacy of various medical and surgical procedures from Williams ${ }^{17}$ (1991 prices)

\begin{tabular}{lc}
\hline Procedure & $\begin{array}{l}\text { Cost } \\
(\mathcal{L} / \text { life-year })\end{array}$ \\
\hline Pacemaker for complete heart block & 1120 \\
Hip replacement & 1200 \\
Valve replacement for aortic stenosis & 1440 \\
Coronary artery bypass grafting for 3 vessel & 2040 \\
$\quad$ disease & 4800 \\
Kidney transplant & 8000 \\
Heart transplant & 17600 \\
Hospital haemodialysis & 19300 \\
$\begin{array}{l}\text { Coronary artery bypass grafting for single } \\
\text { vessel disease }\end{array}$ & \\
\hline (These prices include an element for quality of life and may
\end{tabular}

(These prices include an element for quality of life and

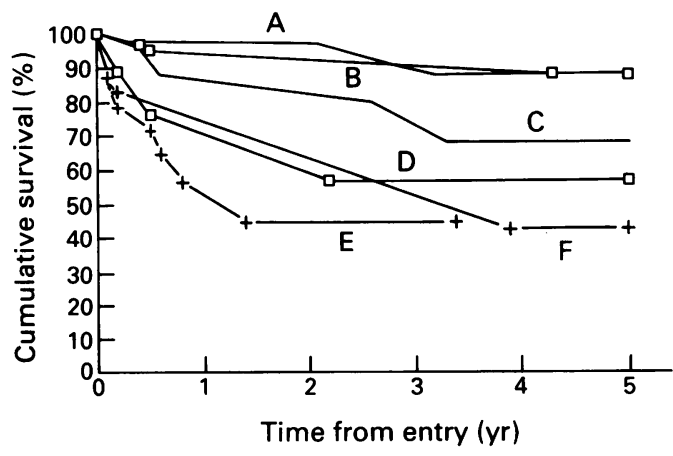

Figure 2 Plot of cumulative survival free of cardiac arrest against time: (group $A$, inducible arrhythmia, suppressed by drugs, high ejection fraction; group $B$, non inducible, high ejection fraction; group $C$, inducible arrhythmia, suppressed by drugs, low ejection fraction; group $D$, non-inducible, low ejection fraction; group $E$, inducible arrhythmia, not suppressed by drugs, low ejection fraction; group $F$, inducible arrhythmia, not suppressed by drugs, high ejection fraction. Modified from Wilber et al $\left.1988^{18}\right)$.

tion and includes a 24 hour stay in a high dependency unit postoperatively. This cost is derived from that used to charge for operations performed on patients from outside our region and includes the cost of staff, equipment, and maintenance of facilities. Our cost is close to that quoted in the study of Buxton, et al. ${ }^{10}$ Similarly the cost of a bed for extra nights is that charged to cover staff, equipment, drugs, and building costs in 1991.

The cost of screening tests and electrophysiological studies have been derived from costs calculated in 1990 and adjusted to 1991 prices to allow for cross charging between the medical school and hospital. All charges include staff time, the cost of equipment depreciated over a two year period, and the costs of ancillary services such as heat and light. Follow up visits have been costed on the basis of a three monthly follow up routine with two further unscheduled visits allowed for in the first year and assuming that $10 \%$ of follow up visits require a day admission for full evaluation of defibrillator function.

Many of the assumptions that we have made about costs may differ in other institutions. A major advantage of our simple model is that it can readily adapt to any alteration of these assumptions.

\section{Results}

APPLICATIONS OF THE COSTS MODEL

We have applied the model to examine three aspects of the cost-efficacy of the use of ICD: (a) the use of an ICD in survivors of out of hospital cardiac arrest; (b) strategies for ICD use proposed by the current generation of controlled trials; $(c)$ the impact of technical and surgical developments on the costefficacy of use of ICDs.

The main purpose of our model is to examine the relative cost-efficacy of various strategies for the use of ICDs. Whereas it is of great interest to know how the cost-efficacy of various uses of ICDs compare with other treatments care must be taken in making such 


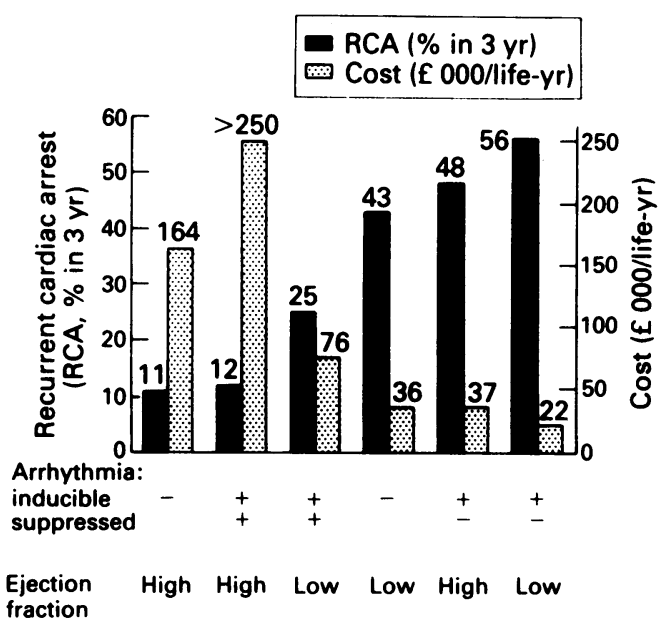

Figure 3 Incidence of recurrent cardiac arrest (RCA) in three years and cost of ICD use in thousand pounds per life-year $(f, 000 / l y)$ in the six groups shown in fig 2. High $>30 \%$; Low $<30 \%$.

comparisons with data from our simple model. The methods used by other investigators are more complex and designed to produce an absolute value for cost-efficacy. They often include an adjustment for quality of life and economic discounting of future years of survival. For general comparison table 3 shows the cost of other medical treatments assessed by Williams in $1985^{17}$ and adjusted for inflation to 1991 prices.

Cost of the use of the ICD in survivors of cardiac arrest

Survivors of an out of hospital cardiac arrest occurring in the absence of acute myocardial infarction are a heterogenous group. Wilber $e t$ al described the long-term outcome when this group is subdivided on the basis of electrophysiological testing combined with measurement of left ventricular ejection fraction. ${ }^{18} \mathrm{He}$ divided patients into subgroups based on whether they had high $(>30 \%)$ or low $(<30 \%)$ left ventricular ejection fraction, whether arrhythmias were inducible at electrophysiological study, and whether these arrhythmias were rendered non-inducible by antiarrhythmic drug treatment (fig 2). With our model in combination with the survival data from this study we have calculated the cost-efficacy of the use of ICDs in each of the subgroups, in the whole population, and in various combinations of subgroups described by Wilber et al ${ }^{18}$ (fig 3). The cost-efficacy of the use of ICDs ranges from $£ 22400$ per lifeyear in the highest risk subgroup (ejection fraction $<30 \%$, inducible arrhythmia, not suppressed by antiarrhythmic drug treatment) to nearly $£ 700000$ per life year in patients with inducible arrhythmia suppressed by drugs and with an ejection fraction $>30 \%$. Whereas ICD use in the highest risk subgroup seems very cost-effective, such a strategy will have little impact on the overall incidence of sudden death as only $27 \%$ of incidents of recurrent cardiac arrest occur in this subgroup. By contrast, a policy of general
ICD implantation in all survivors of cardiac arrest is more expensive ( $£ 57000$ per lifeyear) but could potentially prevent all sudden cardiac deaths. Comparison with the cost of other medical treatments (table 3) suggests that this is an expensive strategy.

A combined approach with the ICD in the three subgroups at highest risk of recurrent cardiac arrest $((a)$ inducible non-suppressed low ejection fraction, (b) inducible nonsuppressed high ejection fraction, (c) noninducible low ejection fraction) increases the potential for prevention of sudden death, as $56 \%$ of recurrent cardiac arrests occur in these groups. This increased yield in the combined high risk group can be gained for a marginal rise in cost to $£ 23600$ per life-year.

In the light of the findings of O'Donoghue et al that early ICD implantation is associated with reduced hospital costs, ${ }^{12}$ we have used our model to assess the cost-efficacy of two simpler approaches to selection of patients for ICD implantation, either baseline inducibility of arrhythmias at a single electrophysiological study, or ejection fraction alone. A single electrophysiological study costs $£ 500$ and may involve one or two extra days in hospital. In the study of Wilber et al $79 \%$ of patients had an inducible arrhythmia and $86.2 \%$ of recurrent cardiac arrests occurred in this group. Because of the cost of an electrophysiological study to identify this large group at relatively low risk of sudden death the cost per life-year of this strategy is $£ 57000$. This is identical to the strategy of no screening test at all and seems to offer no advantage over such a policy. By contrast measurement of ejection fraction is cheap and involves no extra hospital stay. The low ejection fraction subgroup comprises $33 \%$ of the population and $52 \%$ of recurrent cardiac arrests occur in this subgroup. The cost-efficacy of ICD use in this subgroup alone is $£ 25500$ per lifeyear. This is a considerable improvement when compared with ICD use in the whole population ( $£ 57000$ per life-year). Despite the shorter hospital stay, however, this approach is less cost-effective than use of the ICD in the combined high risk group described and would prevent a smaller proportion $(52 \% v 56 \%)$ of the total number of sudden deaths.

Our costs model suggests that generalised use of the ICD in all survivors of cardiac arrest is an expensive strategy. The cost-efficacy may be improved by restricting the use of ICDs to subgroups at higher risk of sudden death. The model suggests that a combination of measurement of ejection fraction and electrophysiological assessment is better at identifying a cost-effective high risk subgroup than either of these investigations alone.

A number of studies are in progress to compare ICD use in survivors of cardiac arrest with conventional treatment. The Dutch Prospective Study is specifically considering the issue of cost-efficacy. ${ }^{19}$ The results of such studies should enable a more rational use of the ICD in these patients. 
Whole population $\Xi$ Inducible Inducible, non-suppressed

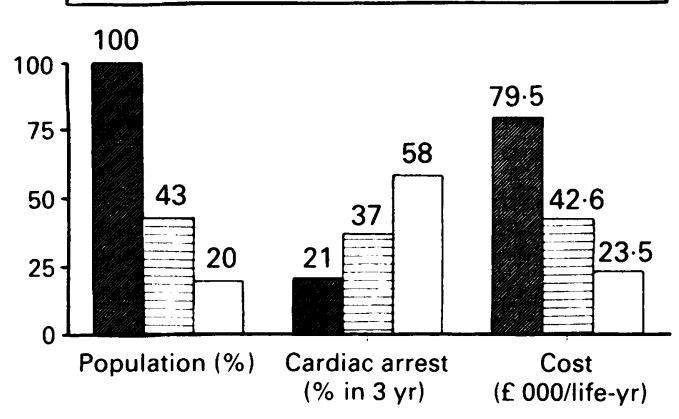

Figure 4 Stratification of patients with non-sustained ventricular tachycardia by the costs model and data from Wilber et al. ${ }^{25}$

\section{Costs of future applications of the ICD}

Several controlled trials of the ICD are currently planned or are under way. ${ }^{2021}$ Among these are some that aim to evaluate new risk groups for implantation of ICDs. These include patients with non-sustained ventricular tachycardia, high risk patients after myocardial infarction, patients undergoing coronary artery bypass grafting, and patients with severe dilated cardiomyopathy who are awaiting cardiac transplantation.

With the costs model we have assessed the cost-efficacy of the strategies proposed by these trials from published survival data for the various groups at risk.

\section{Cost-efficacy of ICDs in patients with non- sustained ventricular tachycardia (NSVT)}

Three separate trials of the use of the ICDs in patients with NSVT and known coronary artery disease but without a history of sustained ventricular tachycardia or ventricular fibrillation are in progress. The MADIT study (Multicenter Automatic Defibrillator Implantation Trial) recruits patients with NSVT who have inducible arrhythmias at baseline electrophysiological study that remain inducible on procainamide. ${ }^{22}$ These patients are randomised to ICD or conventional drug treatment. The MUSTT (Multicenter Unsustained Tachycardia Trial) and SDPS (Sudden Death Prevention Study) both compare electrophysiologically guided drug treatment with placebo in patients with inducible arrhythmia. In the electrophysiologically guided patients the ICD is used for patients who remain inducible despite drug treatment. The rationale for these studies is that the presence of inducible arrhythmias at electrophysiological study correlates closely with subsequent ventricular tachycardia or fibrillation ${ }^{23}{ }^{24}$ and that patients with NSVT have a high incidence of inducible arrhythmias and these patients in turn have a high incidence of sudden death..$^{25} 26$

Wilber et $a l^{25}$ have published data on survival free of cardiac arrest for patients with coronary artery disease, left ventricular ejection fraction $<40 \%$, and non-sustained ven- tricular tachycardia. They have stratified this population by inducibility of sustained ventricular tachycardia at electrophysiological study and whether such tachycardias could be suppressed by antiarrhythmic drug treatment. Figure 4 shows the results of this stratification. With these data and our model the strategy for the use of $\mathrm{ICD}^{5}$ proposed by the MADIT trial costs $£ 42500$ per life-year and the strategy of the MUSTT and SDPS trials cost $£ 23500$ per life-year. This cost-efficacy is similar to that gained with the ICD in survivors of cardiac arrest. Worrying reports of difficulty with recruitment for the MADIT trial $^{27}$ suggest that the prevalence of inducible arrhythmias noted in the study of Wilber et $a l^{18}(43 \%)$ may not be representative of this group as a whole. This would increase the number of patients who need to be screened to find one patient at risk and reduce the cost-efficacy of this strategy.

Cost-efficacy of ICDs in patients at high risk after myocardial infarction

Survivors of myocardial infarction represent a large population who are known to be at increased risk of sudden cardiac death. Much interest has centred on the identification of subgroups of this population who are at particularly high risk. Although electrophysiological studies have been reported to be effective at identifying patients with a high risk of recurrent cardiac events ${ }^{28}$ most interest has focused on the use of non-invasive screening tests. At St George's Hospital we have a large database of survivors of myocardial infarction. Over 500 patients have now been followed up for three years or more, and the population has been studied for predictors of sudden death. Currently the best group of tests to identify patients at high risk seems to be the combination of reduced heart rate variability, more than 10 ventricular extrasystoles an hour, and a positive signal average electrocardiogram. At three years this population has a sudden death rate of $29.9 \%$ in comparison with $4.5 \%$ for the population as a whole and $7.5 \%$ for those patients with an ejection fraction of $<40 \%$ (fig 5 ). The cost of

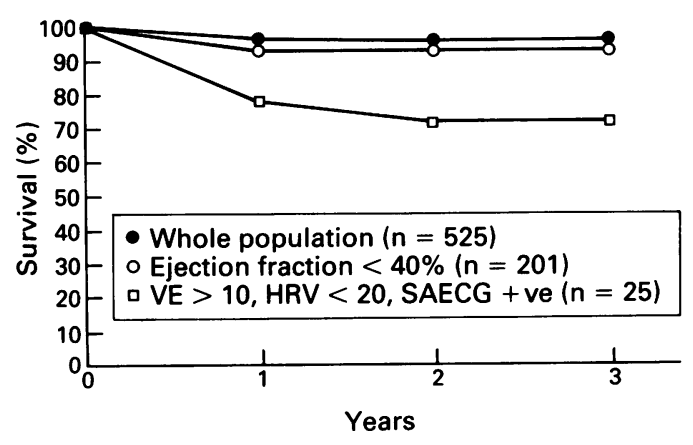

Figure 5 Stratification of survivors of myocardial infarction (data from the St George's Hospital database). $A$ high risk group of patients with frequent ventricular extrasystoles $(V E>10)$, reduced heart rate variability $(H R V<20)$ and positive signal average electrocardiogram (SAECG+ve) a $29.9 \%$ three-year mortality from sudden death. 
ICD use in this high risk population is $£ 36500$ per life-year, which is less than twice the cost when the device is used in the highest risk subgroup of survivors of cardiac arrest. Whereas this group may be approaching an acceptable level of cost-effectiveness it includes only $26 \%$ of the patients who will die suddenly in this period and this represents only $10.5 \%$ of the total deaths. Extension of the use of the ICD to the larger group with an ejection fraction of less than $40 \%$ (38\% of the population) increases the cost nearly fivefold to $£ 170000$ per life-year. The overall impact of ICD use on mortality after infarction is likely to remain small unless the sensitivity and specificity of screening tests for patients at risk of sudden death improves.

The Australasian Clinical Trial of the Automatic Implantable Defibrillator (ACTAID) study that evaluates the implantation of the ICD in patients with inducible arrhythmias after infarction is in the early stages of recruitment and should provide valuable data on the role of the ICD in survivors of myocardial infarction.

\section{Cost-efficacy of ICDs in patients with low ejection fraction and positive signal average electrocardiogram}

The "CABG patch" study is already in progress. ${ }^{29}$ It recruits patients destined for coronary artery bypass grafting (CABG) with impaired left ventricular function (ejection fraction $<35 \%$ ) and a positive signal average electrocardiogram). Patients are randomised to CABG or to CABG plus ICD. Insertion of the ICD at the same time as CABG saves on both surgical and bed stay costs.

There are no published survival data for an identical group, but a retrospective study showed an unexpectedly high mortality. ${ }^{20}$ Data are available for a similar group of patients with a positive signal average electrocardiogram and ejection fraction of $<40 \%$. $^{30}$ The one year mortality in this group is about $12 \%-14 \%$. Extrapolation is required to produce a three year sudden death mortality of $21 \%$ so that the costing figure of $£ 44000$ per life-year is subject to wide confidence intervals. Data from our own database at $\mathrm{St}$ George's Hospital suggest that patients after infarction with these risk factors have a three year sudden death rate of just $4 \cdot 2 \%$. This produces a very high cost of $£ 570000$ per life-year. The patients chosen from our database do not necessarily require coronary artery bypass grafting and might therefore be presumed to be at lower risk of sudden death than those entering the CABG patch study. It is not possible to make any definitive conclusions about the strategy proposed in this trial pending the availability of survival data from the trial itself.

\section{Cost-efficacy of ICDs in patients awaiting cardiac transplantation}

About $40 \%$ of deaths from congestive heart failure are thought to be of an arrhythmic nature ${ }^{31}{ }^{32}$ and patients with dilated cardiomyopathy awaiting cardiac transplantation seem to be at particularly high risk of sudden death. ${ }^{33}$ This has given rise to the suggested use of the ICD as a bridge to transplantation (DEFIBRLAT = defibrillator implantation as bridge to later transplantation ${ }^{34}$ ) although the protocol of this study has yet to be published. Stevenson et al found a $34 \%$ one year sudden death mortality in the group with an ejection fraction $<25 \%$ and the mortality rose to $57 \%$ in those patients with a stroke volume of less than $40 \mathrm{ml}^{33}$ In these patients our costs model was adjusted to assume a one year wait to transplantation with a $50 \%$ five year survival after transplantation. Because of the high mortality and short follow up period in this group the defibrillator seems highly cost is effective $(£ 16000$ per life-year in the whole group and $£ 9300$ per life-year in the high risk group). It must be remembered, however, that these costs are additional to the cost per life-year of the transplant itself.

\section{EXPECTED COSTS OF THE STRATEGIES} PROPOSED BY THE CURRENT GENERATION OF CONTROLLED ICD TRIALS

We have already seen that the cost-efficacy of the various controlled trials of the ICD varies considerably. By calculating the numbers of patients in the various risk groups we can calculate the expected costs for the United Kingdom if the strategies proposed by the trials are adopted. The current population of the United Kingdom is 57 million and the annual incidence of myocardial infarction is estimated at 4 per 1000 of the population. ${ }^{35}$ This gives an annual total of about 225000 of whom about 135000 survive. ${ }^{36}$ The basic cost of each ICD implant alone (without any allowance for screening test or follow up costs) is $£ 16700$.

It is hard to make an accurate assessment of the number of survivors of out of hospital cardiac arrest in the United Kingdom because there is no central registry for such events. A study of out of hospital defibrillation conducted in Scotland would produce a number of just under 4000 if the findings are extrapolated to the whole United Kingdom. ${ }^{37}$ D A figure of 8.3 survivors of cardiac arrest per

Table 4 Costs of adopting the strategies proposed by the various controlled trials of the ICD

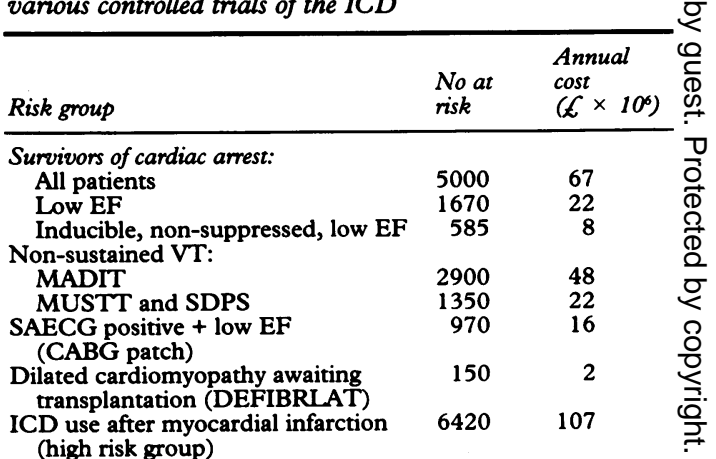

(high risk group)

CABG, coronary artery bypass graft; DEFIBRLAT, defibrillator implantation as bridge to later transplantation; defibrillator implantation as bridge to later transplantation EF, ejection fraction, MADIT, multicenter automatic defibrillator implantation trial; MUSTT, multicenter unsustained tachycardia trial; SDPS, sudden death prev ventricular tachycardia. 


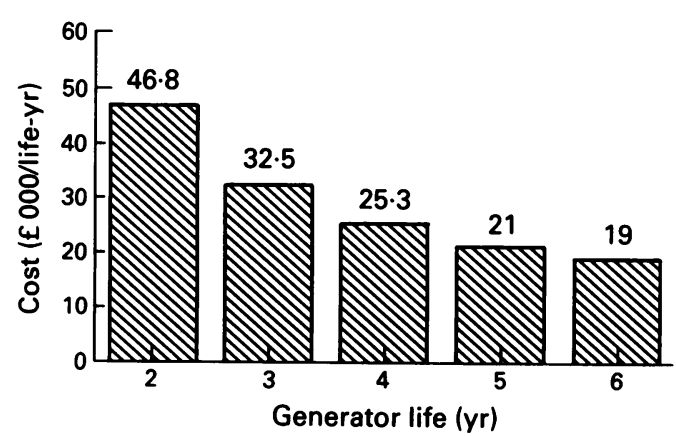

Figure 6 Effect of increasing generator life on the cost-efficacy of ICD use in a hypothetical population with a three year sudden mortality of $28 \%$.

100000 of the population was produced by a study in rural lowa, ${ }^{38}$ and extrapolation to the United Kingdom gives a figure of just under 5000 per year. We have used the figure of 4000 in our calculations. Assuming we give all of these patients an ICD the expenditure required for implantation alone would be $£ 67$ million per year. If we restrict the ICD to those with a low ejection fraction then the cost is reduced to $£ 22$ million per year and if we use the ICD only in the highest risk group with low ejection fraction and inducible non-suppressed arrhythmias the cost falls to $£ 8$ million per year.

In patients with non-sustained ventricular tachycardia we can assume that such patients are selected from the 135000 patients who survive a myocardial infarction. In the $\mathrm{St}$ George's study group $5 \%$ of such patients had non-sustained ventricular tachycardia on Holter monitoring. If we restrict the use of ICDs to the highest risk group with inducible tachycardias (like the MUSTT and SDPS trials) not suppressed by conventional antiarrhythmic drug treatment the yearly cost would be $£ 22$ million. If we adopt the broader strategy of use of the device in all patients with an ejection fraction below $40 \%$ and inducible ventricular arrhythmias (like the MADIT trial) the cost rises to $£ 48$ million a year.

In 1989295 coronary artery bypass operations were performed for every million of the population, a total of 16800 operations. Our own database indicates that $5 \cdot 7 \%$ of these would have a positive signal average electrocardiogram and reduced ejection fraction.

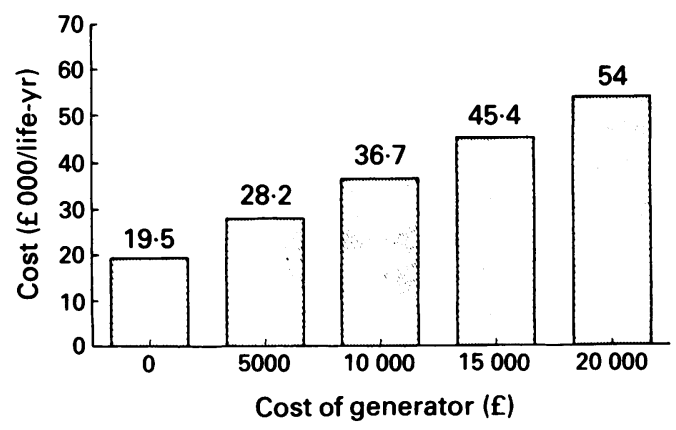

Figure 7 Effect of changing costs of generator on the cost-efficacy of ICD use in survivors of myocardial infarction at high risk of sudden cardiac death.

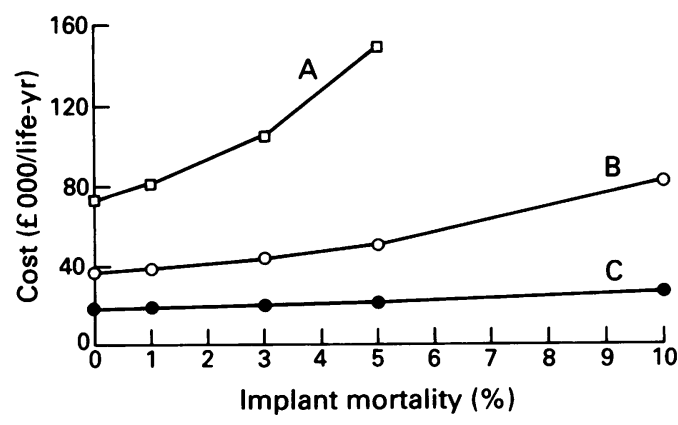

Figure 8 Effect of altering implant mortality on the cost-efficacy of ICD use in three populations with differing three year sudden death rate: $(A), 14 \% ;(B)$, $28 \%$; (C), $56 \%$. The improvement in cost-efficacy from improved implant related mortality is greatest where the use of the ICD is marginal (where sudden death mortality is low).

This would qualify them for the CABG patch trial. If such a policy were adopted in the United Kingdom the yearly cost would be $£ 16$ million.

About 400 heart transplants were performed in the United Kingdom in 1990. If we were to restrict ICD use to the group at highest risk of sudden death (ejection fraction ( $<25 \%$ and stroke volume $<40 \mathrm{ml}$ ) the annual cost would be just $£ 2$ million. By contrast, if the ICD were used in survivors of myocardial infarction the additional expenditure required would be massive. Even to implant only in a selected high risk group (which contains just $5 \%$ of the survivors of infarct) would cost $£ 107$ million a year and have a very limited impact on overall mortality.

All of the strategies proposed by the current generation of ICD trials (with the exception of DEFIBRLAT) have considerable implications for national expenditure on cardiology (table 4). These findings emphasise the need for careful costing to be an integral part of these and future studies of the ICD.

\section{FUTURE TECHNICAL DEVELOPMENTS IN THE} ICD AND SCREENING TESTS

The costs considered so far have been based on a number of assumptions that are likely to change over the next few years. With our costs model we have assessed the impact of

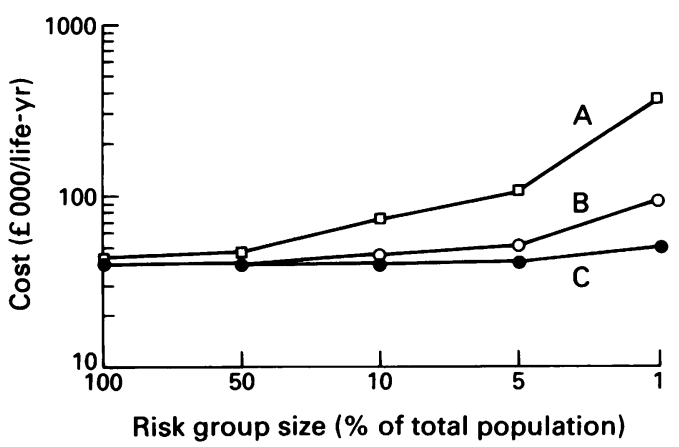

Figure 9 Effect of different screening test costs. (A), $£ 50 ;(B), £ 250 ;(C), £ 1500$ on cost-efficacy in relation to the size of the group selected by the test. The smaller the high risk group selected, the more sensitive it is to cost of screening test. 
these developments in our high risk group after myocardial infarction where the cost per life-year is currently $£ 36500$. Use of the ICD in this group currently seems relatively expensive when compared with other medical treatments.

\section{Increased generator life}

If we assume an increase in the life of a generator to five years and that the sudden deaths are distributed so that two thirds of them occur in the first three years after ICD implantation the cost of ICD use in our high risk postinfarction group falls to $£ 25000$ per life-year. Figure 6 shows the relation between life of the generator and cost-efficacy. Further extensions of life of the generator beyond five years are likely to have only a smaller impact.

\section{Generator price reduced}

With an assumption of a $50 \%$ reduction in the real cost of a simple defibrillator the cost of the use of an ICD in our high risk postinfarction group falls to $£ 28000$ per life-year. Figure 7 shows the relation between generator price and cost-efficacy. It is interesting to note that even if the ICD generator were free the cost of this strategy would still be $£ 19500$ a life-year.

\section{Transvenous implantation}

Transvenous implantation is likely to be associated with a shorter hospital stay and reduced cost of implantation surgery. The cost of the defibrillator, however, is unchanged and most transvenous lead systems are marginally more expensive than their epicardial counterparts. Although transvenous implantation may be expected to improve implantation mortality figures, data currently available have yet to show this effect.

The large series of Medtronic defibrillators showed no deaths among the patients in whom transvenous systems were successfully implanted, but two deaths occurred in patients who went on to have epicardial patch systems. ${ }^{39}$ On an intention to treat basis this does not show any significant improvement over conventional epicardial patches. For this reason we have not assumed any reduction in mortality from implantation and the impact of transvenous implantation alone is small with a reduction in cost of $£ 32000$ per lifeyear. By contrast, figure 8 shows the dramatic improvement in cost-efficacy that could result if mortality from implantation is reduced, particularly in groups in which yearly sudden death rate is relatively small.

\section{Screening tests}

Despite considerable effort improvements in screening tests to identify patients at high risk of sudden death have been slow and are not always reproducible in different centres. The interest in improvements of screening tests is understandable when the economic effects are considered. A screening test able to detect a group with a three year sudden death rate of $60 \%$ would improve cost-efficacy to $£ 18000$ per life-year. Any new screening test must not only be sensitive and specific but should be relatively cheap. Figure 9 shows graphically the relation between the cost of screening tests and the size of the population at risk identified by the tests. It is clear that for a population such as ours, where the risk group represents about $5 \%$ of the population $I$ screened a screening test should not cost more than $£ 250$.

\section{Overall assessment of future costs}

An assessment of the cost of use of an ICD in the future with the cost of a generator halved, five year generator life, transvenous implantation with $1.5 \%$ implant mortality, and improved screening tests would be just $£ 7700$ per life-year, a reduction of over $75 \%$ in the current cost. Changes of this size would have a dramatic effect on the perception of the ICD as an expensive treatment.

\section{Discussion}

Sudden cardiac death remains a public health problem of massive proportion in the western world with probably 70000 sudden deaths a year in the United Kingdom alone. Despite the availability in some communities of cardiopulmonary resuscitation by bystanders and out of hospital defibrillation less than one in three patients suffering a cardiac arrest are resuscitated and survive discharge from hospital. ${ }^{40}$ About two thirds of cardiac arrest patients have some form of cardiac disease recognised before the terminal event and the potential exists to identify those at risk of sudden cardiac death in advance. Until the development of the ICD, however, there was little incentive to do so. The dramatic increase in the frequency of implantation of the $\mathrm{ICD}^{41}$ shows the need for such a device. Initial retrospective studies suggested that the device was very good in preventing sudden cardiac death. 568 This has made the ethics of performing a randomised controlled trial of the use of ICD in survivors of cardiac arrest difficult. No such dilemma, however, affects the study of the prophylactic use of the ICD $\frac{D}{0}$ in patients with no history of cardiac arrest but a high risk of sudden cardiac death in the $\stackrel{N}{\circ}$ future. Hence a large number of studies of $N$ prophylactic use of ICDs are currently $\underset{\mathrm{C}}{N}$ planned or under way.

The expansion in the use of the ICD, however, has not been entirely uncriticised and attention has been drawn to the expense of ? the device. ${ }^{42}$ Similar criticisms were made in the early days of the heart transplantation

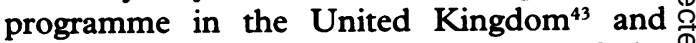
were redressed by a detailed study of the $\stackrel{\mathbb{D}}{\Omega}$ costs and benefits of the technique. ${ }^{44}$

The question of the cost-efficacy of the $\delta$ ICD was first considered by Kupperman et al with decision analysis techniques. ${ }^{9}$ They used data from the 1984 Medicare database, $\stackrel{2}{\rightleftharpoons}$ medical publications, Medicare carriers, individual pharmacies and hospitals, and expert opinion to estimate the cost of ICD treatment versus the cost of conventional treatment in a group of patients with at least 
one episode of cardiac arrest not associated with myocardial infarction. This study concluded that the cost of use of an ICD in survivors of cardiac arrest was well within the range of costs for other life saving interventions in the United States at that time, and that the real cost of ICD treatment would halve by 1991 . The main limitation of this study is the difficulty of knowing exactly how the cost and survival figures were derived. Because of the transatlantic differences between health care systems it is very difficult to know how applicable the findings are to the use of ICDs in the United Kingdom.

Recently Larsen et al have published an analysis that compared the cost-efficacy of amiodarone and ICDs. They used a complex state transition decision model to find the outcome for each population and to calculate the costs incurred. This complex model attempts to account for all possible costs incurred by patients in each group (for example the costs associated with the side effects of amiodarone). The marginal cost-efficacy of amiodarone over conventional treatment was $\$ 6600$ per life-year and the marginal cost-efficacy of the ICD compared with amiodarone was $\$ 29200$ per life-year. Sensitivity analyses were conducted to examine the impact of prolonging generator life and of alterations in the quality of life on amiodarone or ICD treatment. Prolongation of generator life to five years improved ICD cost-efficacy to $\$ 16500$ per life- year. ${ }^{13}$

The cost-efficacy of using the ICD in Britain by comparison with long-term amiodarone treatment was considered by Buston et al. ${ }^{10}$ Their model studied a 20 year period with economic modelling and life expectancy data from a variety of studies. They produced a range of cost-efficacies depending on the survival data used for the amiodarone treated and ICD treated groups. No comparison was made with the cost-efficacy of other accepted medical treatments but the authors again suggested that technical and implantation developments could improve costefficacy two or threefold.

Whereas these three studies are detailed attempts to assess the cost-efficacy of the ICD their complexity makes their usefulness to assess alternative applications of the ICD difficult. To facilitate such comparisons and to enable the impact of various strategies on the cost-efficacy of ICD use to be assessed we have developed a simple model in which the total cost of the use of ICDs is divided by the gain in life-years to produce a cost per lifeyear gained. By the substitution of locally derived costs and with our simple method to calculate the gain in life-years the model may easily be applied to a wide range of different situations. We have applied the model to study the cost-efficacy of some current and possible future indications for the use of ICDs. Unlike the more complex studies described so far our model does not assume any savings in drug treatment and no assumptions are made about subsequent admissions to hospital. Reliable data from large scale studies on these variables are not yet available but there is as yet no evidence that the ICD reduces subsequent costs of drugs or frequency of readmission to hospital.

Our model assumes $100 \%$ prevention of sudden cardiac death by the implantable defibrillator and any deviation from this will increase the cost per life-year saved. Most studies have suggested that the incidence of sudden cardiac death in ICD recipients is very low. ${ }^{6241}$ An adjustment for altered efficacy is easy to include in our model when the gain in life-years is being calculated. A similar adjustment can be performed to assess the effect of varying non-sudden death rates in different groups of patients. High nonsudden death rates also reduce the gain in life-years from the use of ICDs and impair cost-efficacy accordingly.

A recurrent finding during our study has been the trade off that occurs between improving cost-efficacy and reducing the number of sudden deaths prevented. That such a trade off occurs reflects the poor sensitivity and specificity of current screening tests for patients at risk of sudden cardiac death. The model also shows the dramatic improvement in cost-efficacy that can occur if screening tests become more specific. Screening tests must also be cheap enough not to have a significant impact on the cost-efficacy of the use of ICDs in the selected high risk group. Paradoxically, as the high risk group selected by the test becomes smaller the cost of the screening test becomes more and more important in determining overall cost-efficacy of ICD use.

Some trials of the prophylactic use of the ICD are in progress or planned. Our model strongly suggests that the cost-efficacy of all of these strategies lies within a narrow range. This lies only slightly above the range of cost-efficacies for the current widely accepted use of the ICD in survivors of cardiac arrest that in turn is slightly above that of several expensive treatments provided by the NHS. The adoption of any of the prophylactic strategies for ICD use in the United Kingdom would require substantial additional expenditure because of the large number of patients involved.

The assumptions used in the calculation of these costs, however, are rapidly being overtaken by technical improvements, competitive prices, and improved implantation techniques. Combinations of such factors will have a very powerful effect to reduce the cost of the use of ICDs and may result in an improvement in cost-efficacy of up to fivefold over the next few years. This could dramatically alter the perception of the ICD as an expensive treatment.

As more reliable data on the efficacy of the ICD becomes available from prospective randomised controlled trials the findings of this study can be refined. Models of future costefficacy will continue to have important roles for health care planners and in helping to direct future medical research.

In conclusion a flexible model of the cost- 
efficacy of the use of ICDs has been developed and used to examine various strategies and the impact of technical and medical developments. Currently the ICD seems to be an expensive means to prevent sudden death and the cost-efficacy is surprisingly similar for many different applications that are currently being considered for the device. Technical and medical developments are likely to cause a dramatic reduction in the cost of using the device over the next few years but to make a significant impact on the overall incidence of sudden death large numbers of patients will have to be treated and this will need substantial provision of medical and financial resources. Modelling the costefficacy of new medical interventions may prove a useful way to identify those factors that have an important effect on cost-efficacy and those that do not. Careful application of this information may enable more rational provision and use of scarce resources.

1 Mirowski M, Reid PR, Mower MM, et al. Termination of malignant ventricular arrhythmias in man with an implantable automatic defibrillator. $N$ Engl f Med 1980; 303:322-4.

2 Nisam S, Mower M, Moser S. ICD clinical update: first decade, initial 10000 patients. $P A C E$ 1991;14:255-62.

3 Nisam S, Mower MM, Thomas A, Hauser R. Patient survival comparison in three generations of CPI AICD's: review of 12 years, 20000 patients [abstr]. European foumal of Cardiac Pacing and Electrophysiology 1992; 2:A117.

4 Griffith MJ, Varma N, Sowton GE, Rowland E, Perrins EJ, Sutton $R$, et al. Use of automatic implantable defibrillators in the United Kingdom [abstr]. Br Heart $\mathcal{F}$ 1990;64:101.

5 Winkle RA, Mead H, Ruder MA, et al. Long-term outcome with the automatic implantable cardioverterdefibrillator. F Am Coll Cardiol 1989;13:1353-61.

6 Winkle RA, Mead RH, Ruder MA, et al. Ten year experience with implantable defibrillators [abstr] Circulation 1991;84:II-426.

7 Gross J, Zilo P, Ferrick K, Fisher JD, Furman S. Sudden death mortality in implantable cardioverter defibrillator death mortality in implantable

8 Fogoros RN, Fielder SB, Elson JJ. The automatic implantable cardioverter-defibrillator in drug-refractory ventricular

9 Kupperman M, Luce BR, McGovern B, Podrid PJ, Bigger JT, Ruskin JN. An analysis of the cost-effectiveness of the implantable defibrillator. Circulation 1990;81: 91-100.

10 Buxton MJ, O'Brien BJ, Rushby JA. Cost effectiveness of the automatic implantable cardioverter defibrillator:a preliminary assessment. Health Economics Research Group Research Report. London: Brunel University. 1990 Research

11 Herre JM, Sauve MJ, Malone P, et al. Long-term results of amiodarone therapy in patients with recurrent sustained ventricular tachycardia or
f Am Coll Cardiol 1989;13:442-9.

12 O'Donoghue S, Platia EV, Brooks-Robinson S, Mispireta L. Automatic implantable cardioverter-defibrillator: is early implantation cost-effective? $\mathcal{\Im} \mathrm{Am}$ Coll Cardio 1990;16:1258-63.

13 Larsen C, Manolis AS, Sonnenberg FA, Beshansky JR, Mark Estes NA, Pauker SG. Cost-effectiveness of the implantable cardioverter-defibrillator: effect of improved battery life and comparison with amiodarone therapy. 7 Am Coll Cardiol 1992;19:1323-34.

14 Vlay SC, Olson LC, Fricchione GL, Friedman R. Anxiety and anger in patients with ventricular tachyarrhythmias, responses after automatic implantable internal carresponses after automatic implantable internal cardioverter

15 Keren R, Aarons D, Veltri EP. Anxiety and depression in patients with life threatening ventricular arrhythmias: impact of the implantable cardioverter defibrillator. PACE 1991;14:181-7.

16 Kalbfleisch KR, Lehmann MH, Steinman RT, Jackson K, Axtell $\mathrm{K}$, Schuger CD, et al. Reemployment following implantation of the implantable cardioverter defibrillator. Am $₹$ Cardiol 1989;64:199-202.
17 Williams A. Economics of coronary artery bypass grafting. BMF 1985;291:326-9.

18 Wilber J, Garan H, Finkelstein D, Kelley E, Newell J, McGovern B, et al. Out-of-hospital cardiac arrest: use of electrophysiological testing in the prediction of longterm outcome. $N$ Engl f Med 1988;318:19-24.

19 Wever EFD, Hauer RNW. Cost-effectiveness considerations: the Dutch prospective study of the automatic implantable cardioverter defibrillator as first-choice therapy. PACE 1992;15:690-3.

20 Nisam S, Thomas A, Mower $M$, Hauser R. Identifyin patients for prophylactic implantable cardioverter defibrillator therapy: status of prospective studies. Am Hear f 1991;122:607-12.

21 Bigger JT. Future studies with the implantable cardioverter defibrillator. PACE 1991;14:883-9.

22 MADIT Executive Committee: multicenter automatic defibrillator implantation trial (MADIT): design and clinical protocol. PACE 1991;14:920-7.

23 Denniss AR, Richards DA, Cody DV, Russell PA, Yound AA, Ross DL, et al. Prognostic significance of ventricular tachycardia and fibrillation induced at programmed stimulation and delayed potentials detectable on the signal-averaged electrocardiograms of survivors of acute myocardial infarction. Circulation 1986;69:731-45.

24 Richards D, Taylor A, Fahey P, Irwig L, Koo CC, Ross $\mathrm{D}$, et al. Identification of patients at risk of sudden death after myocardial infarction: the continued Australian experience. In: Brugada $\mathrm{P}$, Wellens $\mathrm{H}$, eds. Cardia arrhythmias: where to go from here? Mount Kisco, NY: Futura, 1987:329-41.

25 Wilber DJ, Olshansky B, Moran JF, Scanlon PJ. Electrophysiological testing and nonsustained ventricular tachycardia. Circulation 1990;82;350-8.

26 Kadish A, Schmaltz S, Calkins H, de Buitleir M, Mired F Outcome of patients undergoing electrophysiological testing for non-sustained ventricular tach /cardia [abstr] testing for non-sustaine

27 Multicenter automatic defibrillator implantation trial (MADIT): design and clinical protocol. PACE 1991 14:920-7.

28 Richards DAB, Byth K, Ross DL, Uther JB. What is the best predictor of spontaneous ventricular tachycardia and sudden death after myocardial infarction. Circulation 1991;83:756-63.

29 Bigger JT. Prophylactic use of implantable cardioverter defibrillators: medical, technical, economic considerations. PACE 1991;14:376-80.

30 Gomes JA, Winters SL, Stewart D, Horowitz S, Milne $M$, Barreca $P$. A new noninvasive index to predict sustained ventricular tachycardia and sudden death in the first year after myocardial infarction: based on signalaveraged electrocardiogram, radionuclide ejection fraction and Holter monitoring. $\mathcal{F}$ Am Coll Cardiol 1987, 10:349-57.

31 Packer M. Sudden unexpected death in patients with congestive heart failure: a second frontier. Circulation 1985;72:681-5.

32 Francis G. Should asymptomatic ventricular arrhythmias in patients with congestive heart failure be treated with antiarrhythmia drugs? $\mathcal{F}$ Am Coll Cardiol 1988;12:274-6.

33 Stevenson LW, Fowler MB, Schroeder JS, Stevenson WG, Dracup KA, Ford V. Poor survival of patients considered too well for cardiac transplantation. $\mathrm{Am} \mathcal{F} \mathrm{Med}$ sidered too well

34 Bolling S, Deeb M, Mired F, et al. AICD: a new "bridge" to transplantation [abstr]. $\mathcal{F} \mathrm{Am}$ Coll Cardiol 1990 15:223A.

35 Petch MC. Myocardial infarction. In: Julian DG, Camm AJ, Fox KM, Hall RJC, Poole-Wilson PA, eds. Diseases of the Heart. London:Baillière Tindall, 1989:1157-91.

36 Tunstall-Pedoe $\mathrm{H}$, Clayton $\mathrm{D}$, Morris $\mathrm{JN}$, Brigden W, McDonald L. Coronary heart attacks in East London. Lancet 1975;2:833-8.

37 Cobbe SM, Redmond MJ, Watson JM, Hollingworth J, Carrington J. "Heartstart Scotland"-initial experience of a national scheme for out of hospital defibrillation BMf 1991;302:1517-20.

38 Stults KR, Brown DD, Schug VL, Bean JA. Prehospital defibrillation performed by emergency medical technidefibrillation performed by emergency medical 1984 cians in rural

39 Lindemans FW, van Binsbergen E, Connolly D. European $P C D^{T M}$ study patients with Transvene ${ }^{T M}$ lead systems Maastricht: Medtronic Bakken Research Centre, 1991.

40 Weaver WD, Cobb LA, Hallstrom AP, Fahrenbruch C, Copass MK, Ray $\mathbf{R}$. Factors influencing survival after out-of-hospital cardiac arrest. $\mathcal{F}$ Am Coll Cardiol 1986; 7:752-7.

41 Nisam S, Mower M, Moser S. ICD clinical update: First decade, initial 10000 patients. PACE 1991;14:255-62.

42 Campbell RWF. Life at a price: the implantable defibrill tor [editorial]. Br Heart f 1990;64:171-3.

43 O'Brien BJ, Buxton MJ, Ferguson BA. Measuring the effectiveness of heart transplantation programmes:quality of life data and their relationship to survival analysis. ty Chron Dis 1987;40:137S-53S.

44 Buxton MJ, Acheson R, Caine N, Gibson S, O'Brien BJ. Costs and benefits of the heart transplant programmes at Costs and benefits of the heart transplant programmes at
Harefield and Papworth hospitals. DHSS Research Report. London:HMSO, 1985 (No.12). 